\title{
Intraoperative localisation of the primary motor cortex using single electrical stimuli
}

\author{
A Maertens de Noordhout, J D Born, P Hans, J M Remacle, P J Delwaide
}

Department of

Neurology

A Maertens de

Noordhout

P J Delwaide

Department of

Neurosurgery

J D Born

J M Remacle

Department of

Anaesthesiology,

Hôpital de la Citadelle,

4000 Liège, Belgium

P Hans

Correspondence to:

Dr A Maertens de

Noordhout, University

Department of Neurology,

Hôpital de la Citadelle, 4000

Liège, Belgium.

Received 5 June 1995

and in final revised form

5 October 1995

Accepted 8 November 1995

\begin{abstract}
A new method of intraoperative localisation of the primary motor cortex is described, based on the application of single anodal electric pulses to the brain surface. Patients were anaesthetised with propofol infusion, and neuromuscular blockade was temporarily alleviated to allow recording of surface EMG responses (CMAPs) to the stimuli. Primary motor areas could be localised in $18 / 19$ patients studied. In the other patient, no responses were elicited, as the operative field was posterior to the motor cortex. When compared with MEPs elicited in awake patients by magnetic stimuli, responses to intraoperative anodal stimulation were of small amplitude (usually less than $10 \%$ of MEPs) and their latency was some 1 to 2 ms longer. One month after the operation, only 1/19 patients was left with a slight muscle weakness, although seven showed preoperative motor deficit. The procedure proved easy and fast, needing no preliminary surgery or time consuming preparation. It did not induce any detectable side effects.
\end{abstract}

$(F$ Neurol Neurosurg Psychiatry 1996;60:442-444)

Keywords: motor cortex localisation; single electrical stimuli

ered to the brain. Moreover, it does not allow quantitative measurements of the responses elicited. Other techniques have been proposed $^{34}$ but they are either invasive or time consuming.

We report a novel method of intraoperative localisation of cortical motor areas, using single anodal electrical stimulations applied to the brain surface and recordings of the EMG activity elicited in contralateral muscles. As it was developed for routine use in tumour surgery, it had to be safe and easy to perform, needing no time consuming preparation or preliminary surgery.

\section{Patients and methods}

PATIENTS

After approval by the local ethics committee, localisation of the motor cortex was undertaken in 19 patients during resection of intrinsic brain tumours located near primary motor areas, as determined by CT or MRI. Motor evoked potentials (MEPs) to transcranial magnetic stimulations were recorded before surgery, to evaluate the functional state of corticospinal pathways and to avoid false negative results of intraoperative recordings. Patients were anaesthetised with intravenous infusion of propofol and sufentanyl. Before motor cortex localisation, atracurium infusion was temporarily interrupted to restore satisfactory neuromuscular transmission.

\section{ELECTROMYOGRAPHIC RECORDINGS AND}

\section{STIMULATIONS}

Muscle twitches elicited by single stimuli were of the brain carries the risk of damaging functionally important structures. Despite progress in neuroimaging the limits of the primary motor cortex remain difficult to delineate. Location of the Rolandic sulcus shows between individual variability and the normal brain architecture can be distorted by the tumour or surrounding oedema. ${ }^{1}$ Therefore, intraoperative electrophysiological techniques have been proposed to localise motor cortical areas with precision. Usually, trains of electric shocks $(50$ or $60 \mathrm{~Hz}, \mathrm{AC})$ are applied to the cortical surface with visual inspection of the twitches elicited in contralateral muscles. ${ }^{2}$ When this procedure is performed under general anaesthesia, its safety can be questioned with respect to the amount of current deliv- brief and less readily detectable by visual inspection than with trains of stimulations. For this reason, and to allow precise localisation of the optimal stimulation sites, we recorded EMG activity (CMAPs) with adhesive surface electrodes placed over appropriate muscles. Responses were amplified $(50 \mu \mathrm{V} / \mathrm{V})$ and filtered $(20 \mathrm{~Hz}-5 \mathrm{kHz})$, then stored for further analysis. The present method was not designed to draw a detailed map of motor cortical areas but to localise the motor strip for tumour surgery. Therefore, in most instances, CMAPs were simultaneously recorded in four muscles, chosen in function to show the presumed localisation of the brain lesion. For a given position of the stimulating anode, four 
Mean latencies (ms) and peak to peak amplitudes ( $m V$ ) of EMG responses recorded in different muscles to preoperative magnetic and intraoperative electric stimulation of the motor cortex

\begin{tabular}{lll}
\hline Muscle & $\begin{array}{l}\text { Latency (magneticlelectric) } \\
(\mathrm{ms})\end{array}$ & $\begin{array}{l}\text { Amplitude (magneticlelectric) } \\
(\mathrm{mV})\end{array}$ \\
\hline Orbicularis oris $(\mathrm{n}=7)$ & $11 \cdot 4 / 13 \cdot 5$ & $1 \cdot 7 / 0 \cdot 13$ \\
Deltoid $(\mathrm{n}=6)$ & $11 \cdot 4 / 12 \cdot 9$ & $2 \cdot 9 / 0 \cdot 16$ \\
Biceps $(\mathrm{n}=13)$ & $12 \cdot 9 / 14 \cdot 4$ & $2 \cdot 4 / 0 \cdot 26$ \\
Extensor digitorum $(\mathrm{n}=15)$ & $17 \cdot 1 / 19 \cdot 2$ & $2 \cdot 9 / 0 \cdot 18$ \\
Thenar muscles $(\mathrm{n}=7)$ & $22 \cdot 2 / 24 \cdot 1$ & $5 \cdot 1 / 0 \cdot 27$ \\
FDI (n = 16) & $22 \cdot 7 / 24 \cdot 4$ & $4 \cdot 9 / 0 \cdot 32$ \\
Quadriceps $(\mathrm{n}=2)$ & $22 \cdot 8 / 25 \cdot 3$ & $1 \cdot 5 / 0 \cdot 22$ \\
Tibialis anterior $(\mathrm{n}=2)$ & $30 \cdot 7 / 32 \cdot 4$ & $2 \cdot 1 / 0 \cdot 21$ \\
\hline
\end{tabular}

Mean latency difference $1.7 \mathrm{~ms}$; Mean amplitude ratio (electric:magnetic) $8 \cdot 4 \%$. enhanced CT of patient 19 showing a left rolandic glioblastoma causing generalised seizures and weakness of the right upper limb. Motor examination of the patient seven days after the operation was normal.
Figure 1 Preoperative

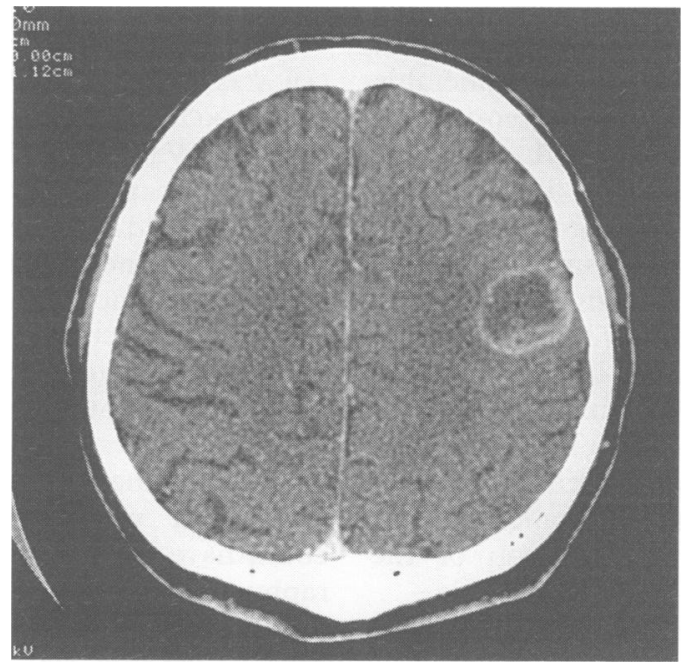

to eight EMG responses were recorded and superimposed to measure their amplitude and latency. The motor cortex was stimulated with a conventional EMG constant current stimulator (Nicolet Viking I, Madison Wisconsin). The stimulating cathode (reference) was a 6 $\mathrm{cm}^{2}$ steel plate fixed sagittally on the forehead with tape. When recording from facial mus-

Figure 2 Maximal CMAPs recorded intraoperatively in three different muscles of patient 19. Four responses are superimposed on each trace. Although of small amplitude, CMAPs are reproducible for a given anode position (stimulus intensities $30 \mathrm{~mA}$ for biceps, $22 \mathrm{~mA}$ for extensor digitorum, and $18 \mathrm{~mA}$ for thenar muscles).
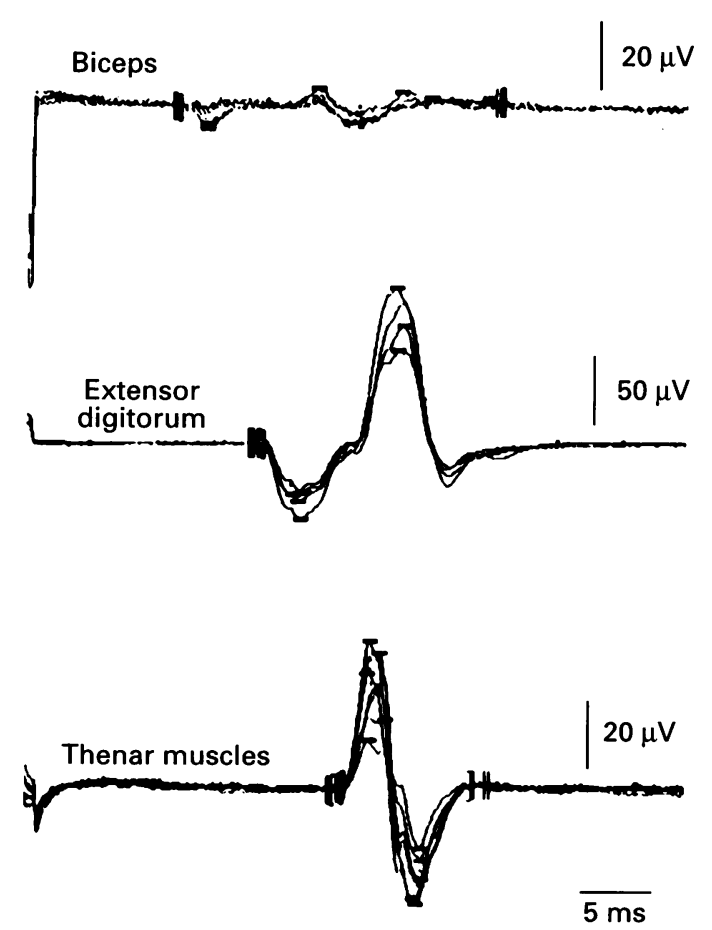

cles, the stimulating cathode was placed in the occipital region, to avoid direct activation of head and face muscles by the stimulus. The stimulating anode (active) was an insulated stainless steel rod embedded in a perspex handle with a rounded tip providing a contact surface of $4 \mathrm{~mm}^{2}$. Motor cortex localisation was undertaken after opening the dura mater. Stimulus characteristics were the following: single square waves of $1 \mathrm{~ms}$ duration, intensities from 10 to $40 \mathrm{~mA}$. Between 30 and 50 stimulations were usually sufficient to localise accurately the motor area of a given muscle. In two patients, muscle responses evoked by single anodal pulses were compared with those produced by conventional bipolar $50 \mathrm{~Hz}$ train stimulations (current intensity 5-15 mA, 0.2 ms square pulses, train duration $2 \mathrm{~s}$, interelectrode distance $4 \mathrm{~mm}$ ). For each muscle, the optimal stimulation site was determined as the anode position eliciting responses of maximal amplitude (stimulus intensity $40 \mathrm{~mA}$ ). After this site had been determined, stimulus intensity was reduced to $10 \mathrm{~mA}$ above threshold for the optimal site and mapping of the motor area of the target muscle was completed. This arrangement was chosen as in preliminary experiments, no further increase of CMAP amplitude was noted at the optimal stimulation site above this stimulus intensity.

\section{Results}

MOTOR CORTEX LOCALISATION

In all patients but one, reproducible CMAPs could be recorded intraoperatively in one or several muscles tested in response to single anodal cortical stimulations. For upper limb muscles, CMAPs could not be evoked if the stimulating anode was displaced more than 10 $\mathrm{mm}$ away from its optimal position. In two patients, the optimal anode position was not anterior, but just posterior to the Rolandic fissure for hand and forearm muscles. In two patients, responses were elicited in leg muscles from stimulations of broader cortical areas, up to $25 \mathrm{~mm}$ anterior and $15 \mathrm{~mm}$ posterior to the Rolandic fissure. The actual localisation of primary motor cortical areas differed from what was predicted by radiographs and bony landmarks in eight of 19 patients.

\section{CHARACTERISTICS OF EMG RESPONSES}

Responses recorded intraoperatively were of smaller amplitude and slightly longer latency than MEPs evoked preoperatively in awake patients by transcranial magnetic stimulations. Table 1 summarises characteristics of responses recorded in various muscles. Figures 1 and 2 present an illustrative case. In six patients, reproducible CMAPs, although of small amplitude, could be recorded in some muscles in the absence of visible muscle twitches. In two patients, the effects of single anodal pulses and $50 \mathrm{~Hz}$ train stimulations were compared. In both, the former elicited responses from a more restricted zone. With $50 \mathrm{~Hz}$ stimuli, twitches could be sometimes evoked as far as $1.5-2 \mathrm{~cm}$ away from the optimal position, where no detectable CMAPs 
were obtained with anodal pulses. This was particularly the case when bipolar stimulating electrodes were oriented parallel to the Rolandic fissure.

\section{FUNCTIONAL OUTCOME AFTER SURGERY AND} SIDE EFFECTS

In all cases, macroscopically complete removal of the lesion was possible, while sparing primary motor areas. Seven patients presented with some motor deficit before the operation. In three of them we noted a transient postoperative worsening of hand and arm weakness which subsided in two after two to five days. One month after the operation, all but one showed normal strength and dexterity of the affected hand. The last patient was left with mild hand clumsiness but with considerable improvement compared with the preoperative state. The other four patients with preoperative deficit showed immediate improvement and function of the affected limbs was fully restored after one month. Among the 12 patients with normal preoperative motor function, only one showed transient (two weeks in duration) weakness of the left hand. We found no seizures or other detectable side effects. Duration of the operation was not prolonged by more than 15 minutes and temporary alleviation of neuromuscular blockade did not cause any problem for the surgeon or the anaesthesist.

\section{Discussion \\ ADVANTAGES OF THE PRESENT METHOD OF CORTICAL LOCALISATION}

Our method of intraoperative localisation of the motor cortex proved safe and easy to perform. It only required placement of the stimulating and recording electrodes and can be performed with every commercially available EMG apparatus, without the need of costly equipment or preliminary surgery. Anaesthesia was not modified, except for temporary reduction of neuromuscular blockade. With single stimuli, the total amount of current delivered to the brain is far less than with trains of stimuli. Recording EMG activity in target muscles seemed useful, because on several occasions CMAPs were detected in the absence of obvious muscle twitches. Moreover, most body parts are usually inaccessible to visual inspection during such operations. The number of muscles in which responses were recorded was limited, as the present method was not designed to draw detailed maps of motor areas, but simply to localise the motor strip for surgery. In two patients, comparison between single anodal pulses and conventional train stimulations indicated that the first allowed a more precise localisation of motor areas despite the use of higher current intensities for individual stimuli. Indeed, it has been shown in animals that brief monopolar anodal pulses applied to the cortex surface have a more focal effect than bipolar stimuli. ${ }^{5}$

CHARACTERISTICS OF EMG RESPONSES

Electromyographic responses elicited by single anodal stimuli were highly reproducible for a given anode position. However, intraoperative CMAPs were of much smaller amplitude and slightly longer latency than MEPs evoked by magnetic transcranial stimulation in awake patients. This is likely due to the combined effects of propofol anaesthesia and of residual neuromuscular blockade. Propofol, like many anaesthetic compounds, reduces cortical and anterior horn cell excitability and can even abolish MEPs to magnetic stimuli. ${ }^{67}$ Whereas magnetic stimuli mostly activate cell bodies or inteneurons, electrical anodal pulses directly excite pyramidal axons which are less sensitive to cortical depression induced by anaesthetic compounds.

\section{LOCALISATION OF MOTOR CORTICAL AREAS AND FUNCTIONAL OUTCOME}

In 18 of 19 patients, the primary motor cortex could be localised. In the other patient, the operative field and the lesion were largely posterior to the motor strip. In eight patients, the actual localisation of motor areas differed from what was expected from MRI and bony landmarks, as already noted by others. ${ }^{2}$ In two patients, the optimal anode position for hand motor areas was located posterior instead of anterior to the Rolandic fissure. Both had a subcortical prerolandic mass lesion which could have mechanically distorted the topography of the motor cortex. ${ }^{4}$ Localisation of leg motor areas was less precise and required stronger stimuli (40-50 mA) than for upper limbs, probably due to their deep location in the interhemispheric fissure, which renders their access uneasy. When the 19 patients were evaluated clinically one month after surgery, only one exhibited a slight motor deficit, although there was some preoperative motor dysfunction in seven patients. Definite conclusions about the usefulness of this procedure cannot be drawn from this work, as there was no matched control group. However, we retrospectively analysed the clinical records of the last 20 patients treated for similar lesions by the same neurosurgeons without electrophysiological monitoring. Of these, six (30\%) were left with a noticeable and permanent motor deficit (severe in two, moderate in four).

1 Kido D, LeMay M, Levison A, Benson W. Computed tomographic localization of the precentral gyrus. Radiology 1980;135:373-7.

2 Ebeling U, Schmid UD, Ying H, Reulen HJ. Safe surgery of lesions near the motor cortex using intra-operative mapping techniques: a report on 50 patients. Acta Neurochir (Wien) 1992;119:23-8.

3 Wood CC, Spencer DD, Allison T, McCarthy G, Williamson PD, Goff WR. Localization of human sensorimotor cortex during surgery by cortical surface recording 68:99-111.

4 Uematsu S, Lesser RP, Fisher RS, et al. Motor and sensory cortex in humans: topography studied with chronic subdural stimulation. Neurosurgery 1992;31:59-71.

5 Phillips CG, Porter R. Unifocal and bifocal stimulation of the motor cortex. F Physiol (Lond) 1962;162:532-8.

6 Jellinek DA, Jewkes D, Symon L. Noninvasive monitoring of intraoperative motor evoked potentials under propofol anesthesia: effects of spinal surgery on motor evoked potential amplitude and latency. Neurosurgery 1991;29. 551-7.

7 Taniguchi $M$, Nadstawek J, Langenbach U, Bremer $F$, Schramm J. Effects of four anesthetic agents on moto evoked potentials elicited by magnetic transcranial stimulation. Neurosurgery 1993;33:407-15. 